\title{
Inferring spatial distribution of the radially integrated transmissivity from pumping tests in heterogeneous confined aquifers
}

\author{
Nadim K. Copty, ${ }^{1}$ Paolo Trinchero, ${ }^{2}$ and Xavier Sanchez-Vila ${ }^{3}$ \\ Received 18 August 2010; revised 28 December 2010; accepted 26 January 2011; published 20 May 2011.
}

[1] Hydrologists routinely analyze pumping test data using conventional interpretation methods that are based on the assumption of homogeneity and that, consequently, yield single estimates of representative flow parameters. However, natural subsurface formations are intrinsically heterogeneous, and hence, the flow parameters influencing the drawdown vary as the cone of depression expands in time. In this paper a novel procedure for the analysis of pumping tests in heterogeneous confined aquifers is developed. We assume that a given heterogeneous aquifer can be represented by a homogeneous system whose flow parameters evolve in time as the pumping test progresses. At any point in time, the interpreted flow parameters are estimated using the ratio of the drawdown and its derivative observed at that particular time. The procedure is repeated for all times, yielding timedependent estimates of transmissivity $T_{i}(t)$ and storativity, $S_{i}(t)$. Based on the analysis of the sensitivity of drawdown to inhomogeneities in the $T$ field, the time-dependent interpreted transmissivity values are found to be a good estimate of $T_{g}(r)$, the geometric mean of the transmissivity values encompassed within a progressively increasing radius $r$ from the well. The procedure is illustrated for Gaussian heterogeneous fields with $\ln (T)$ variances up to a value of 2 . The impact of the separation distance between the pumping well and observation point on data interpretation is discussed. The results show that information about the spatial variability of the transmissivity field can be inferred from time-drawdown data collected at a single observation point.

Citation: Copty, N. K., P. Trinchero, and X. Sanchez-Vila (2011), Inferring spatial distribution of the radially integrated transmissivity from pumping tests in heterogeneous confined aquifers, Water Resour. Res., 47, W05526, doi:10.1029/2010WR009877.

\section{Introduction}

[2] Accurate definition of subsurface flow parameters is an essential step in any hydrogeological study. A common approach for the estimation of flow parameters is through the interpretation of pumping tests. Early, yet still widely used, pumping test interpretation techniques include the Theis method [Theis, 1935] and Cooper-Jacob method [Cooper and Jacob, 1946] for confined aquifers, and the Hantush inflection point method [Hantush, 1956] and the graphical Walton method [Walton, 1962] for leaky aquifers. All these methods assume that the subsurface system can be represented by one or at most a few homogeneous units. However, in reality natural subsurface systems are heterogeneous and, hence, their representative hydraulic parameters are spatially variable and support-dependent. Given the significance of aquifer heterogeneity on groundwater flow and contaminant transport, considerable effort

\footnotetext{
${ }^{1}$ Institute of Environmental Sciences, Bogazici University, Istanbul, Turkey.

${ }^{2}$ AMPHOS 21 Consulting S.L., Barcelona, Spain.

${ }^{3}$ Department of Geotechnical Engineering and Geosciences, Universitat Politecnica de Catalunya-BarcelonaTECH, Barcelona, Spain.
}

Copyright 2011 by the American Geophysical Union. 0043-1397/11/2010WR009877 has been devoted to the estimation of representative flow parameters (e.g., the review by Sanchez-Vila et al. [2006]).

[3] Over the past three decades, different approaches have been proposed for the parameter estimation problem. One approach has been to formulate a formal inverse problem whereby measurements of the state variable (hydraulic head) and prior information are used to estimate flow parameters over the entire domain [e.g., Carrera and Neuman, 1986; McLaughlin and Townley, 1996; de Marsily et al., 1999; Sun and Yeh, 2007]. The main difficulty of such an approach is that the inverse problem can be ill-posed as a result of data scarcity and noise [Sun, 1994]. To alleviate problems resulting from the scarcity of direct hydrological data, some authors have proposed the incorporation of related secondary data into identification of the spatial distribution of flow parameters, such as tracer test data [Bellin and Rubin, 2004; Huang et al., 2004; Ptak et al., 2004] and geophysical data [Rubin and Hubbard, 2005; Slater, 2007; Finsterle and Kowalsky, 2008].

[4] Novel field data acquisition techniques that can potentially enhance the characterization of aquifer heterogeneity have been proposed recently. One such technique is hydraulic tomography, which involves sequential pumping or injection from one of a series of wells and monitoring head change in the other wells [e.g., Gottlieb and Dietrich, 1995; Butler et al., 1999; Bohling et al., 2002; 
Yeh and Liu, 2000; Zhu and Yeh, 2005; Illman et al., 2007; Fienen et al., 2008; Castagna and Bellin, 2009; Yin and Illman, 2009]. The densely collected head/discharge data are then used in an inverse procedure to identify the underlying spatial structure of the parameters. Another example is the application of direct-push (DP) technologies, such as DP slug tests, DP injection logging, and the hydraulic profiling tool. These technologies involve the advancement of the DP tool through shallow subsurface for rapid delineation of hydrogeological structures and estimation of their hydraulic properties [Butler et al., 2002, 2007; Dietrich and Leven, 2005; Dietrich et al., 2008; Köber et al., 2009].

[5] Nonetheless, in many cases the only available information consists of drawdown data at a few observation points obtained from intermediate to long-time pumping tests. Since hydraulic tests are quite expensive, there is a need to maximize information derived from each individual test. For this reason, in recent years there has been renewed interest in reevaluating the information obtained from the application of pumping test interpretation procedures to heterogeneous aquifer systems. For example, Meier et al. [1998] and Sanchez-Vila et al. [1999] showed that the transmissivity $T$ estimated from drawdown data from heterogeneous confined aquifers using the CooperJacob method is close to the geometric mean and insensitive to the location of the observation point. Contrarily,

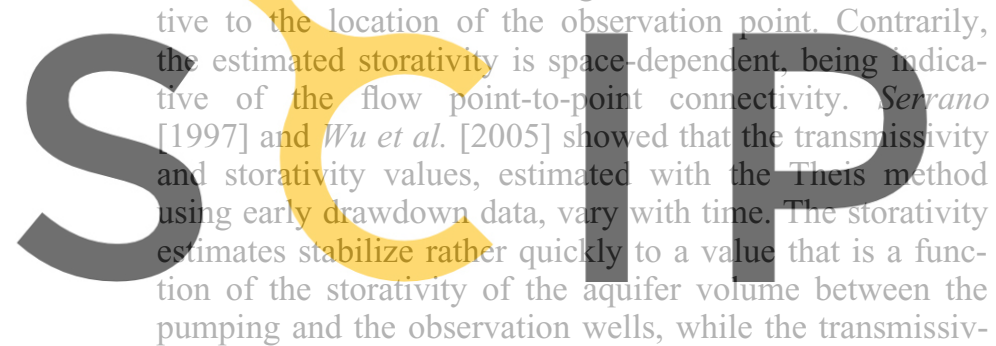

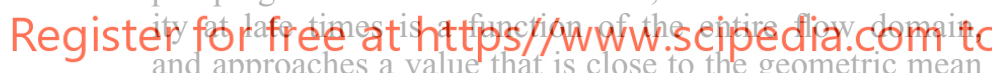
[supporting the results of Sanchez-Vila et al. 1999]. Oliver [1993] used the Frechet kernel to evaluate numerically the sensitivity of variation of the transmissivity and storativity on the transient drawdown as a result of pumping. Knight and Kluitenberg [2005] extended the work of Oliver [1993] by deriving analytic expressions of the Frechet kernels for pumping and slug tests. Leven and Dietrich [2006] used sensitivity coefficients to study the effect of spatial variability of the transmissivity and storativity of a confined aquifer on the interpretation of single-well and two-well pumping tests. For leaky aquifer systems, Copty et al. [2008] compared estimated flow parameters from standard analysis procedures developed for homogenous media when applied to pumping tests conducted in heterogeneous ones.

[6] Several studies have examined the potential use of drawdown data to estimate directly the statistical spatial structure of hydraulic conductivity or transmissivity rather than actual local values. The spatial structure is defined in terms of a few parameters, such as the integral scale and variance of local $T$ values [Copty and Findikakis, 2004a, 2004b; Neuman et al., 2004, 2007; Firmani et al., 2006; Riva et al., 2009]. This group of methods shows that the spatial structure of the flow parameters may be estimated from drawdown data, provided a large number of observation wells are available.

\section{Motivation}

[7] Despite the significance of heterogeneity for groundwater flow and contaminant transport and the significant efforts that have been directed toward this problem, field hydrogeologists often rely on the application of conventional pumping test analysis methods to interpret field data, disregarding the presence of underlying heterogeneity. Such methods use drawdown data collected at different times to estimate representative values of the flow parameters. For example, graphical methods [e.g., Theis, 1935; Walton, 1962] attempt to fit drawdown data observed at all available times to normalized drawdown curves. The Cooper-Jacob method, instead, is based on fitting a straight line to the late drawdown data on a semilog plot. For homogeneous aquifers, the different interpretation techniques yield estimates of flow parameters that are equal to actual transmissivity and storativity values of the aquifer. However, when applied to heterogeneous aquifers, the estimates (termed "interpreted parameters" by Sanchez-Vila et al. [2006]) obtained from conventional interpretation methods vary as the drawdown cone of depression expands in time. For instance, at early times interpreted transmissivity should be close to the $T$ value at the well, since the drawdown signal extends to
only a small region surroundimg the well. At later timos, a
larger aquifer volume is pertrber by the primping tes/ ard,
hence, the estimated transmissivity approximates some
weighted average of the $T$ valyes surrounding the well. The
spatially variable we ghts depend on the relative location of
the pumping and observation points, the statistical spatial
structure of the transmissivity field, leakage if present, the dimensionality of the problem, the imposed boundary con-

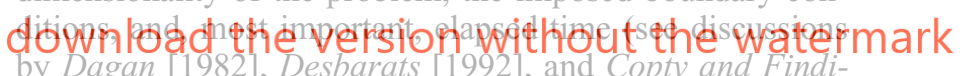
kakis [2004b]). Furthermore, estimated parameters also depend on the interpretation technique used, since each method emphasizes different portions of the time-drawdown data (see Trinchero et al. [2008a] for a discussion on this topic for the specific case of leaky aquifers).

[8] Another limitation of some of the existing methods (e.g., the Cooper-Jacob method for confined aquifers and the Hantush inflection method for leaky aquifers) is that, although they are straightforward and simple to apply, they use only a subset of the available information. In the Cooper-Jacob method only the late drawdown data are used, while the Hantush inflection method uses the steady state drawdown and information at the inflection point only. Although the use of just a portion of the drawdown curve is justified when the aquifer is homogeneous, in the case of a heterogeneous system, this means that important information provided by portions of the time-drawdown data are completely disregarded.

[9] In this paper we develop a simple procedure for analysis of pumping tests in heterogeneous confined aquifers that addresses some of the above limitations and that can yield information about the variability of $T$ in space in a fast and cost-efficient way. The method uses drawdown data and derivatives at one particular point in time to 
estimate flow parameters. It is shown that the interpreted transmissivity is related to how the spatially integrated $T$ values around the well change with distance from the well.

\section{Mathematical Derivation}

\subsection{Estimation of Time-Dependent Flow Parameters}

[10] We consider radially convergent transient flow toward a well in a heterogeneous confined aquifer. We further assume that the shallow approximation or the Dupuit assumption is valid, which implies that vertical flow is negligible [Dagan et al., 2009]. A consequence of the Dupuit simplification is that local transmissivity, defined as the integral of hydraulic conductivity over depth, becomes a formation property that is independent of flow [Sanchez-Vila et al., 2006; Dagan et al., 2009].

[11] For a fully penetrating well in a homogeneous nonleaky confined aquifer, the transient drawdown is given by [Theis, 1935]

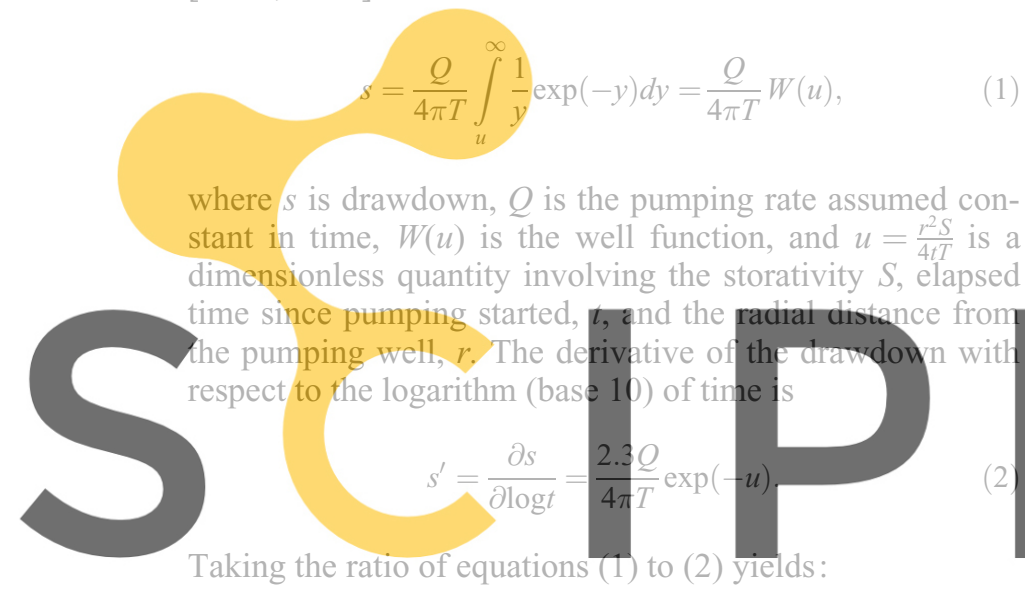

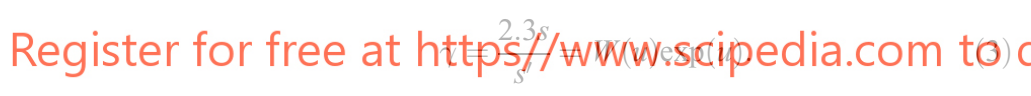

[12] A plot of $\gamma$ as a function of $1 / u$ is given in Figure 1, showing that $\gamma$ increases monotonically with increase in $1 / u$ (i.e., increase in time). Therefore, for any particular value of $\gamma$ at some time $t$, one can uniquely determine the value $u^{*}$ and, consequently the well function $W\left(u^{*}\right)$. Then, the interpreted transmissivity $T_{i}$ and storativity $S_{i}$ can be computed from equation (1) and the definition of $u$, respectively,

$$
\begin{gathered}
T_{i}=\frac{Q}{4 \pi s(t)} W\left(u^{*}\right) \\
S_{i}=\frac{4 t T_{i} u^{*}}{r^{2}} .
\end{gathered}
$$

[13] Equations (3)-(5) provide a simple and fast way to obtain estimates of flow parameters from the ratio of drawdown to the drawdown derivative $\gamma$. The advantage of using the drawdown derivative in the estimation of flow parameters is that the derivative is more sensitive to heterogeneity (as is also discussed in section 3.2.1).

[14] For noise-free drawdown data as a result of pumping in a homogeneous confined aquifer, the above equations would yield the exact values of flow parameters regardless of time and location. However, for pumping tests conducted in heterogeneous (real) aquifers, equations (4) and (5) yield interpreted estimates of transmissivity and storativity that are, in general, dependent on time. By repeatedly applying equations (4) and (5) to different elapsed times, plots of the interpreted time-dependent transmissivity $T_{i}(t)$ and storativity $S_{i}(t)$ are obtained.

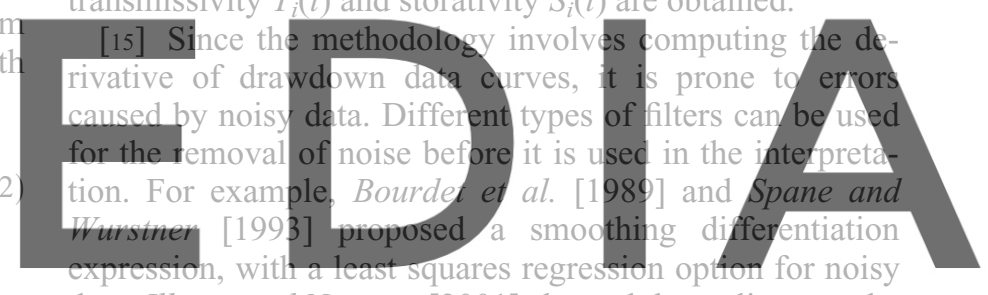

data. Illman and Neuman [2001] showed that splines can be

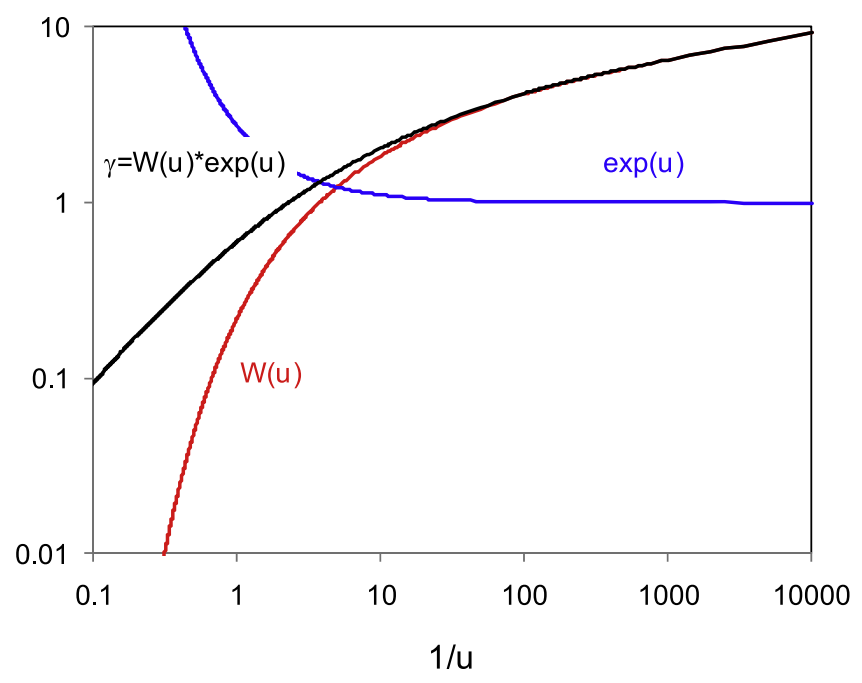

Figure 1. Plot of $\gamma$, equation (3) as a function of $1 / u$. 
examining the sensitivity of the drawdown and its time derivative to the spatial distribution of inhomogeneities in the transmissivity field. Using a perturbation expansion, the transient drawdown at an observation point $\left(r_{o}, \theta_{o}\right)$ as a result of pumping from a heterogeneous aquifer can be expressed as follows:

$$
s\left(r_{o}, \theta_{o}, t\right)=s^{(0)}\left(r_{o}, t\right)+s^{(1)}\left(r_{o}, \theta_{o}, t\right)+s^{(2)}\left(r_{o}, \theta_{o}, t\right)+\cdots,
$$

where $s^{(0)}\left(r_{o}, t\right)$ is the drawdown of the equivalent (homogeneous) aquifer and the other terms, $s^{(j)}\left(r_{o}, \theta_{o}, t\right)$ for $j=$ $1, \ldots$ are solutions of stochastic partial differential equations that describe deviation from the homogeneous solution obtained from the perturbation expansion of the flow equation. Assuming that storativity is constant, the firstorder term of equation (6) can be written as follows:

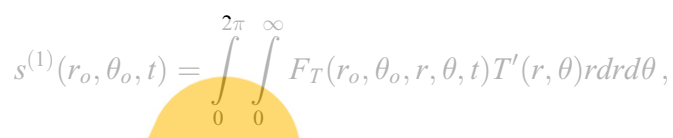

where $F_{T}\left(r_{o}, \theta_{o}, r, \theta, t\right)$ is the Frechet kernel for transmissivity [Oliver, 1993; Parker, 1994] and $T^{\prime}(r, \theta)$ is the deviation of the transmissivity from its representative mean value (e.g., the geometric mean if $T$ is assumed logGaussian and isotropic). $s^{(1)}\left(r_{o}, \theta_{0}, t\right)$ represents the transient variation in the drawdown at the well due to deviation of transmissivity from its mean value. Equation (7) can be viewed as a convolution integral with the Frechet kernel acting as a space and time-dependent weighting function,

The corresponding impact of heterogeneity of transssivity on the time derivative of the drawdown is given by

Register for foee at https//WWNW.Scipeditaldom (to

[18] We consider a homogeneous field $T_{\mathrm{o}}$ and a single anomaly with transmissivity, $T_{o}+T$, located at $(r, \theta)$. If we associate the anomaly to a unitary support volume ( $\Delta V$, actually defined in 2D) and neglect higher-order perturbation terms (i.e., retaining only the first-order terms in the perturbation expansion), we can express the drawdown and its time derivative at the observation well as follows:

$$
\begin{array}{r}
s\left(r_{o}, \theta_{o}, t\right)=s^{(0)}\left(r_{o}, t\right)+F_{T}\left(r_{o}, \theta_{o}, r, \theta, t\right) T^{\prime}(r, \theta) \Delta V \\
\frac{\partial s\left(r_{o}, \theta_{o}, t\right)}{\partial t}=\frac{\partial s^{(0)}\left(r_{o}, t\right)}{\partial t}+\frac{\partial F_{T}\left(r_{o}, \theta_{o}, r, \theta, t\right)}{\partial t} T^{\prime}(r, \theta) \Delta V .
\end{array}
$$

[19] From equation (10) it can be seen that the time derivative of the Frechet kernel is directly related to deviations of $\frac{\partial s\left(r_{o}, \theta_{o}, t\right)}{\partial t}$ from the homogeneous value. In other words, the time derivative of the Frechet kernel is an indicator of the time-dependent influence of anomalous transmissivity on the time derivative of the drawdown at the observation point.

\subsubsection{Observation Point Coinciding With the Pumping Well}

[20] For the case of an observation point coinciding with the pumping well and 2-D flow in a confined aquifer, the Frechet kernel is given by [Knight and Kluitenberg, 2005]

$$
F_{T}(r, t)=-\frac{Q}{8 \pi^{2} T_{o}^{2} D t} \exp \left(-\frac{r^{2}}{2 D t}\right) K_{1}\left(\frac{r^{2}}{2 D t}\right),
$$

where $D$ is the diffusivity equal to $T_{o} / S$ and $K_{1}$ denotes the modified Bessel function of the second kind, of order one. Introducing a scaling parameter $C=-\frac{Q}{2 \pi^{2} T_{o}^{2} r^{2}}$ and the nondimensional time $\frac{1}{u}=\frac{4 T_{o} t}{r^{2} S}$ yields the dimensionless Frechet $\operatorname{kernel}\left(F_{T}^{*}=F_{T} / C\right)$

$$
F_{T}^{*}(1 / u)=u \exp (-2 u) K_{1}(2 u)
$$

[21] Figure 2 shows the plot of the dimensionless Frechet kernel $F_{T}^{*}(1 / u)$ and its derivative with respect to the dimensionless time, $\frac{\partial F_{T}^{*}(1 / u)}{\partial(1 / u)}$. It can be noticed that the impact of the point anomaly on drawdown extends for the entire duration of the pump test. Moreover, $F_{T}^{*}(1 / u) \rightarrow 1 / 2$ as $1 / u$ $\rightarrow \infty$, or equivalently $F_{T}(r, t) \rightarrow-\frac{Q}{4 \pi^{2} T^{2} r^{2}}$ as $t \rightarrow \infty$. This shows that at large times the impact of the anomaly involved in equation (8), which eventually is integrated in $r$, approaches a finite limit that is proportional to $1 / r^{2}$ (where $r$ is the location of the anomaly relative to the
pumping well, which coincides with the observation point).
$[22]$ On the other hand, the influence of the point andm-
aly on $\frac{\partial F_{\pi}^{*}(1 / u)}{\partial(1 / u)}$ and the time derivative of the drawdown is
limited to a relatively short period of time located around
the peak value of equation (12). A similar observation was made by Oliver [1993]. The location of the peak value can be found analytically by setting the second time derivative dementead the version without the watermark

$$
\frac{\partial^{2} F_{T}^{*}(1 / u)}{\partial(1 / u)^{2}}=0
$$

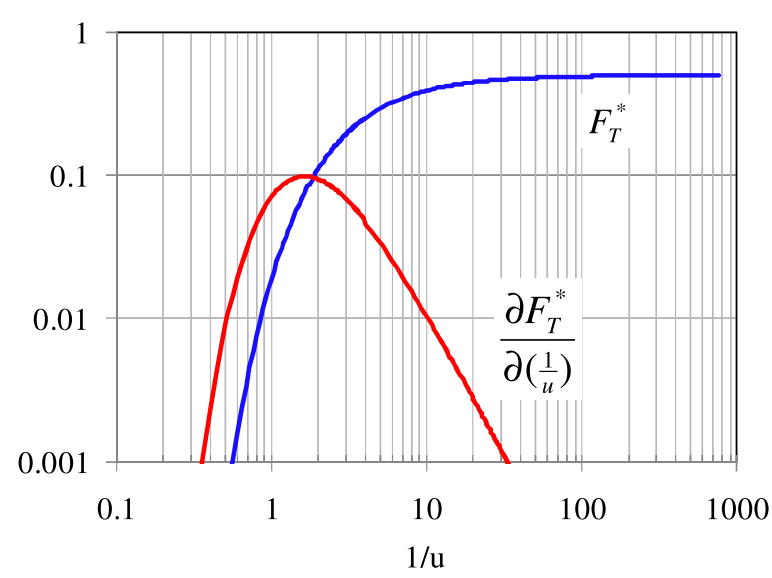

Figure 2. Dimensionless Frechet kernel and its time derivative for the case of coinciding pumping well and observation point. 
[23] It can be shown mathematically that this is equivalent to

$$
\frac{1}{2} K_{o}(2 u)+(1-2 u)\left[K_{o}(2 u)+K_{1}(2 u)\right]=0,
$$

where $K_{0}$ is the modified Bessel functions of the second kind, of order 0 . The root of equation (14) is given by $1 / u=1.6505$.

[24] The physical interpretation of this result is that when pumping commences, a pressure signal is initiated at the well and this signal travels radially outwards. The dimensionless characteristic time $1 / u=1.6505$ is the time needed for the peak of the pressure anomaly (defined as the point corresponding to maximum $\left.\frac{\partial s^{(1)}}{\partial t}\right)$ to travel to the anomaly and back, a total distance of $2 r$.

\subsubsection{General Case (Observation Point Not} Coinciding With the Pumping Well)

[25] In the general case, when the observation point and the pumping well are not coinciding, the dimensionless Frechet kernel is given by [Knight and Kluitenberg, 2005]
$F_{T}^{*}(X, Y, \tau)=-\frac{R^{2}-1 / 4}{8 \pi^{2} R_{1} R_{2} \tau} \exp \left(-\frac{R^{2}+1 / 4}{2 \tau}\right) K_{1}\left(\frac{R_{1} R_{2}}{2 \tau}\right)$.

[26] All distances in equation (15) are normalized by $a$, the separation distance between the pumping well and the observation point. $X$ and $\mathrm{Y}$ are the dimensionless coordinates of the point where the anomaly in located. $R$ is the dimensionless distance between the anomaly and the origin, which, without loss of generality, is located midway between the observation and pumping well. $R_{1}$ and $R_{2}$ are the dimensionless distances from the anomaly to the pumping and observation wells, respectively. $\tau=\frac{D t}{r_{0}^{2}}$ is the dimensionless elapsed time.

[27] Figures $3 \mathrm{a}$ and $3 \mathrm{~b}$ show the derivative of the dimensionless Frechet kernel as a function of dimensionless time for an anomaly located at different distances $R$ and along two directions: (1) along the line passing through the two wells and (2) perpendicular to the line joining the two wells. Whereas in the case of coinciding pumping well and observation point, the travel distance of the pressure signal to the $T$ anomaly and back is $2 r$, in the case of noncoinciding
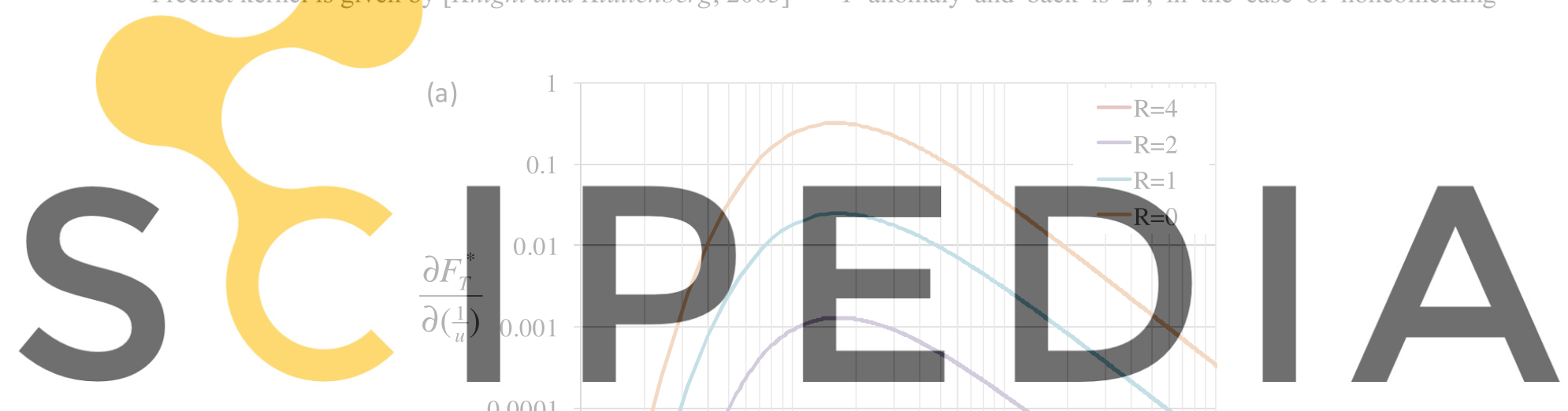

Register for free at https//www.scipedia.com to download the version without the watermark 1E-05

0.1

10

100

$1 / u$

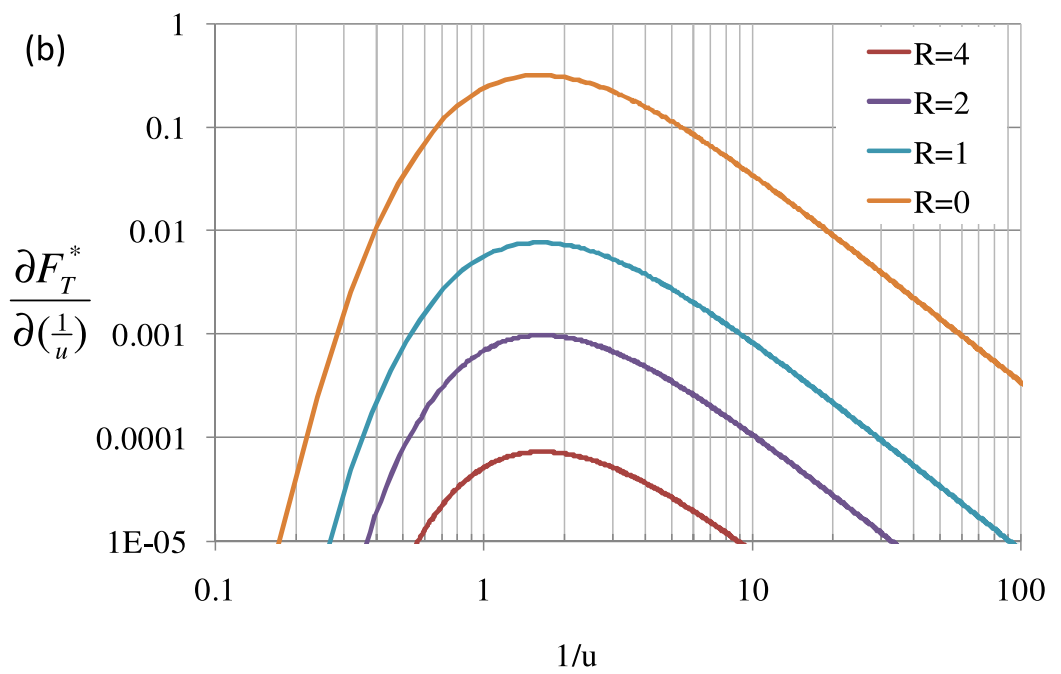

Figure 3. Time derivative of the dimensionless Frechet kernel for an anomaly in the transmissivity field at different distances and either (a) along or (b) perpendicular to the direction joining the pumping and observation points. 
pumping and observation points, the corresponding travel distance of the anomalous signal is $R_{1}+R_{2}$; thus, the length dimension in the $x$ axis of Figure 3 is expressed as $\left(R_{1}+R_{2}\right) / 2$. Figure 3 shows that for both directions considered and irrespective of where the anomaly and observation point are located, the dimensionless Frechet kernel peaks at about the same dimensionless time, $1 / u=1.65$. Using this characteristic dimensionless time, the interpreted transmissivity at time $t, T_{i}(t)$, can be converted to a radially dependent function $T_{i}\left(r^{*}\right)$ where

$$
r^{*}=\sqrt{\frac{4 t T}{1.65 S}} .
$$

[28] In other words, after estimating $T_{i}(t)$ and $S_{i}(t)$ from the drawdown data at any particular time $t$ using equations (4) and (5), equation (16) can be used to map the time $t$ into a radial distance $r^{*}$. In the following section, the proposed pumping test interpretation methodology will be demonstrated using synthetically simulated pumping tests in randomly generated heterogeneous confined aquifer systems.

\section{Numerical Applications}

[29] In the following set of simulations, we consider $Y=$ $\ln (T)$ fields that are realizations of a multivariate Gaussian spatial random function with an exponential semivariogram trith mean $m_{Y}=0$ (i.e., $T_{g}=1$ ), integral seale $I-8$ arbi-
Transmissivity fields were generated using the Turning
Bands Method [Mantoglou and $\left.\boldsymbol{W}_{\text {illson, }}, 982\right]$. The domain
size is 481 by 481 length units, and the grid is uniform with
elements of size 1 by 1 . Storativity is assumed uniform in
space with a value equal to $10^{-4}$. A well is placed at the center of the aquifer and a pumping test is simulated using MODFLOW [Harbaugh et al., 2000]. Transient drawdown

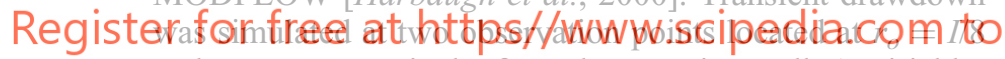
and $r_{o}=I$, respectively, from the pumping well. A Dirichlet condition was imposed at the outer boundary. Preliminary simulations were performed to assure that for the duration of the pumping test, the drawdown was not impacted by the prescribed outer boundary conditions.

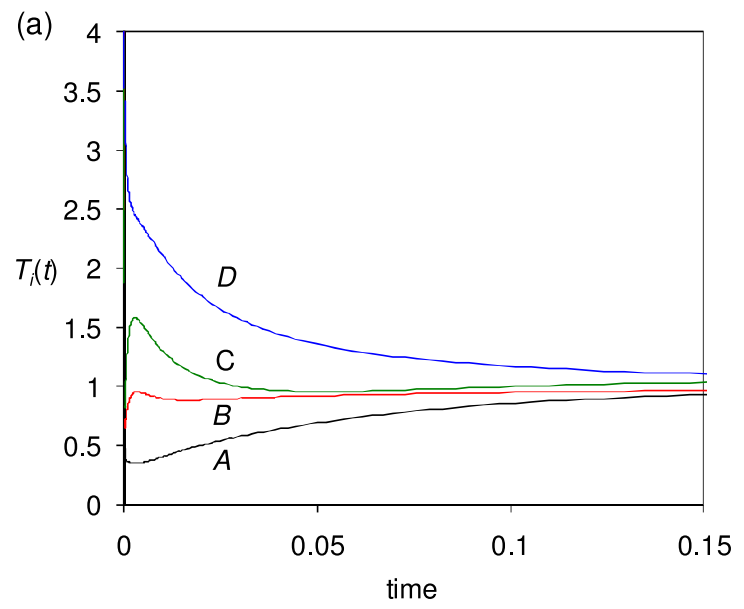

[30] The evolution of drawdown at the observation points was then used to estimate the time-dependent transmissivity and storativity using equations (4) and (5). The very late time drawdown values, which would be influenced by the prescribed condition at the outer boundary of the domain, were excluded from analysis.

\subsection{Estimation of Time-Dependent Transmissivity and Storativity}

[31] The analysis procedure is first applied to four randomly selected $\ln (T)$ fields with variance, $\sigma_{Y}^{2}=1$. Figure $4 \mathrm{a}$ shows the plot of the interpreted transmissivity $T_{i}(t)$ as a function of time for each of these four transmissivity fields based on drawdown data observed at $r_{o}=I / 8$. At early times, large variations between the different estimates are observed because drawdown is influenced by transmissivity in the vicinity of the well, which varies from one realization to the other depending on the value of the log-transmissivity variance. As the pumping test progresses in time, a larger aquifer volume influences the pumping test and as a result $T_{i}(t)$ approaches the geometric mean of the generated $T$ field. Figure $4 \mathrm{~b}$ shows $T_{g}(r)$, the geometric mean of the point $T$ values within a distance $r$ from the well, which was computed as follows:

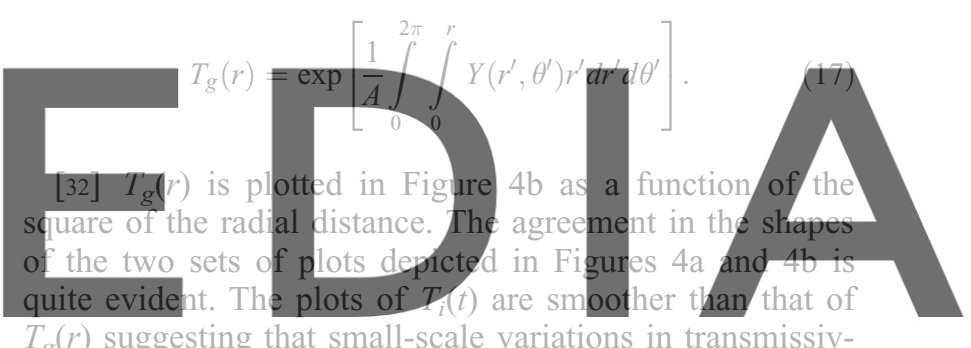
$T_{g}(r)$ suggesting that small-scale variations in transmissivity cannot be fully detected.

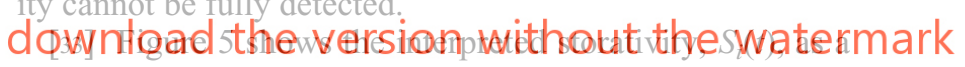
function of time computed using equation (5), corresponding to the four $T$ fields. Although storativity was assumed to be uniform in the pumping test simulation, $S_{i}(t)$ exhibits large variations in time and between realizations. Inspection of Figure 4 and Figure 5 jointly shows that the

(b)

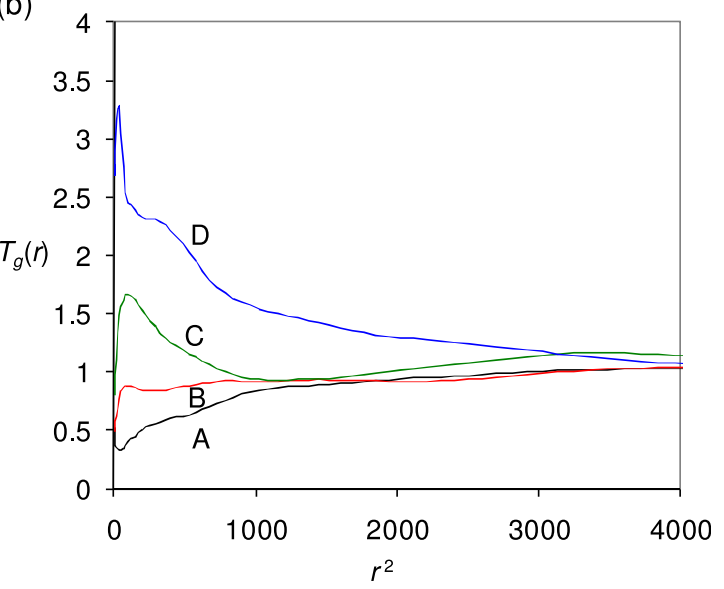

Figure 4. (a) Interpreted transmissivity as a function of time, and (b) Geometric mean as a function of radial distance squared; for four randomly selected $T$ fields. The observation point is located at $r_{o}=I / 8$. 


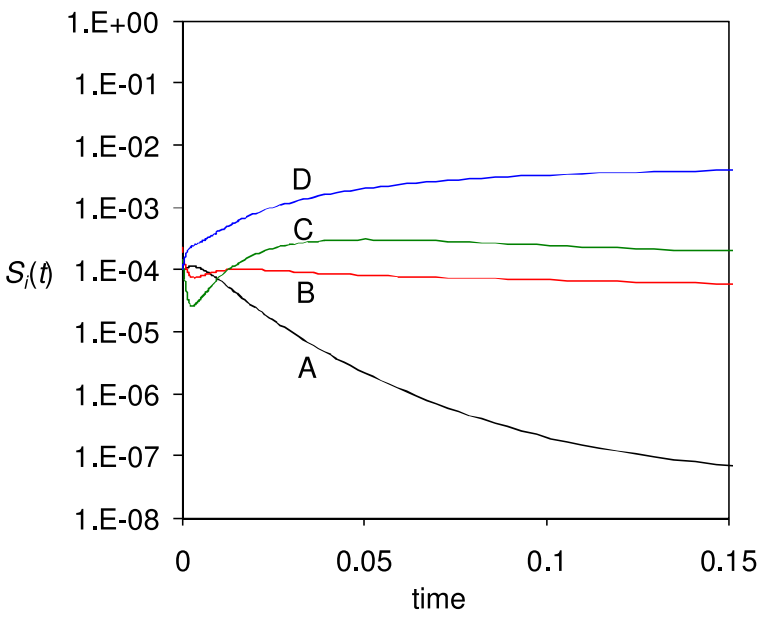

Figure 5. Internreted storativity as a function of time for four randomly selected $T$ fields. The actual storativity value used in the drawdown simulation is 0.0001 .

interpreted transmissivity, $T_{i}(t)$, and storativity, $S_{i}(t)$ are negatively correlated: when $T_{i}(t)$ decreases with time, $S_{i}(t)$ increases, eventually becoming larger than the actual $S$ value and vice versa. This is consistent with previous theoretical studies [Sanchez-Vila et al., 1999; Trinchero et al., 2008b, Fernàndez-Garcia et at, 2010] that showed analytically that storativity estimates point-to-point connectivity the transmissivity field Whereas these studies show the impact of point-to-poin connectivity on the estimation of a single representative storativity value, the results presented herc extend this findIng to the entire transient behavi or of the system.

[34] A significant side result from Figure 5 is that at early times, the cone of depression has sampled a limited

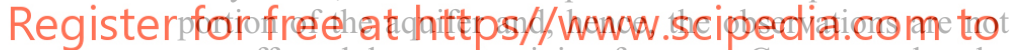
yet affected by connectivity features. Consequently, the interpreted storativity values are relatively close to the real value. In real applications, however, estimation of storativity is error prone since the interpreted values are also affected by a number of processes such as well storage or well development, among others.

\subsection{Comparison of Interpreted Transmissivity to the Radial Geometric Mean}

[35] Equation (16) allows for conversion of the timedependent transmissivity estimates $T_{i}(t)$ to radially dependent averages $T_{g}(r)$. Figure 6 shows the corresponding plot of $T_{i}(r)$ as a function of $r$ estimated from drawdown data observed at $r_{o}=I / 8$. Each of the curves is superimposed on the geometric mean of the four transmissivity fields $T_{g}(r)$ (i.e., Figure $4 b$ ). The good agreement between the four pairs of curves indicates that interpreted transmissivity estimated from the ratio of the drawdown to drawdown rate according to equation (4) is a good indicator of the geometric mean of transmissivity values defined over a continuously increasing circle centered at the pumping well. This is so despite the fact that the time-dependent support volume of transmissivity for radially convergent flow in heterogeneous aquifers is not strictly circular.

[36] The pumping test analysis procedure is now repeated for each of the four randomly selected fields but

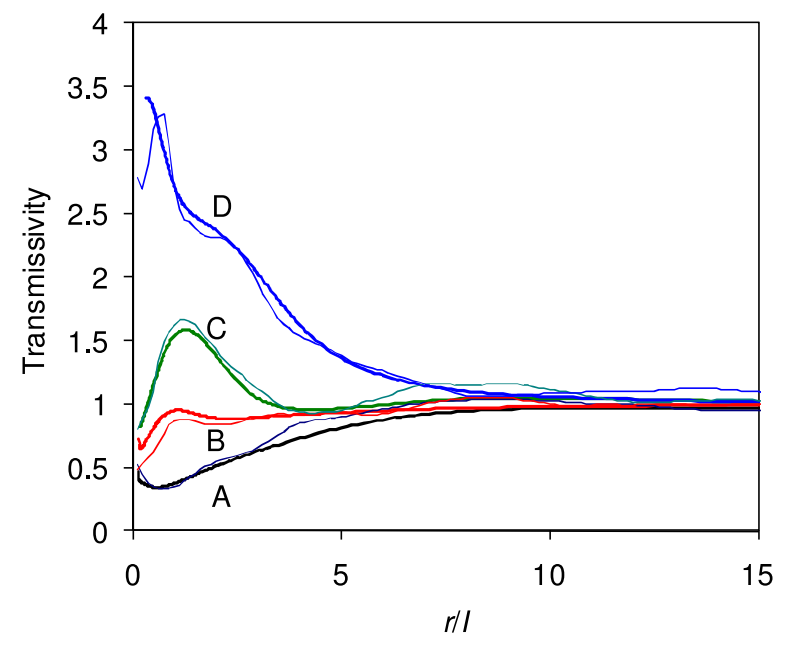

Figure 6. Interpreted transmissivity (in bold) and the geometric mean of the transmissivity (thin line) as a function of radial distance (normalized by the integral scale) for the four randomly selected $T$ fields. The observation point is located at $r_{o}=I / 8$.

using drawdown data from an observation point at $r_{o}=I$. Figure 7 shows the corresponding plot of $T_{i}(r)$ as a function of $r$, which was computed using equation (16). As in Figure

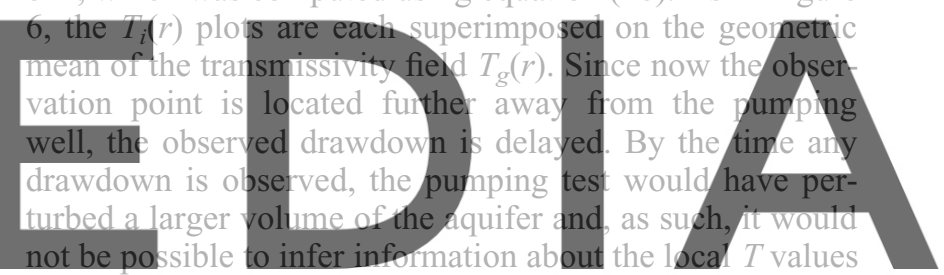

near the pumping well. For observation points located further away such that $r_{0} \gg I$, the drawdown data would be

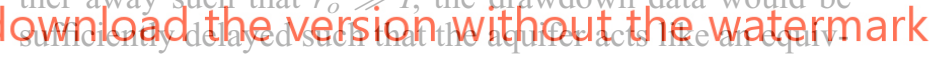
alent homogeneous aquifer and the interpretation would yield virtually uniform $T_{i}(r)$ estimates. Hence, the proposed

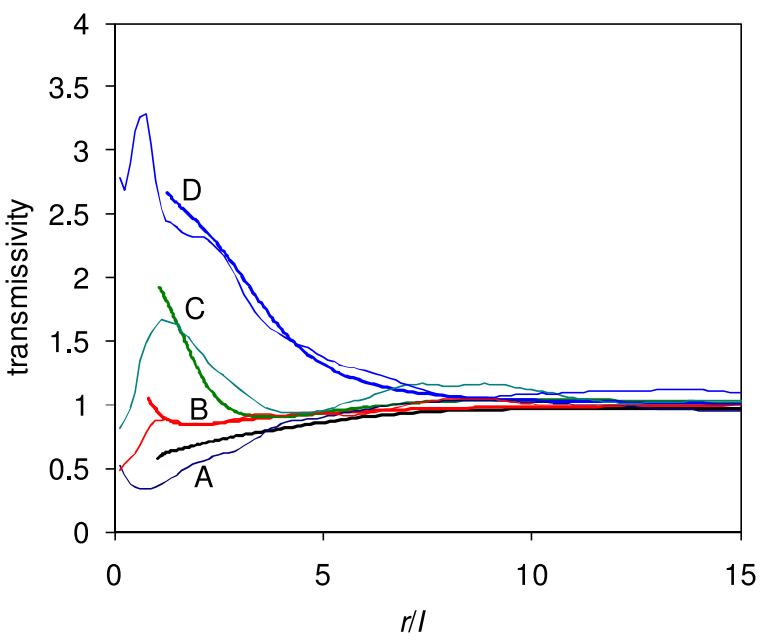

Figure 7. Interpreted transmissivity (in bold) and the geometric mean of the transmissivity (thin line) as a function of radial distance (normalized by the integral scale) for the four randomly selected $T$ fields. The observation point is located at $r_{o}=I$. 
method would potentially yield the most information when the observation well is located at a distance from the pumping well, which is small compared to the integral scale of the $T$ spatial field. In practice this is not a significant limitation because the distances between the pumping well and observation points are normally smaller than the integral scale of the transmissivity field, which ranges from tens to hundreds and even thousands of meters [Gelhar, 1993].

\subsection{Monte Carlo Simulations}

[37] In the preceding section the proposed pumping test interpretation method was applied to a few selected transmissivity fields. In this section we use Monte Carlo simulations to evaluate the performance of the interpretation method over an ensemble of simulations. The model setup is similar to that defined in the previous section. The $\ln (T)$ field is assumed to be multivariate Gaussian with exponential semivariogram and variance $\sigma_{Y}^{2}$ ranging from 1 to 2 . For each set of parameters, 200 transmissivity fields are randomly generated.

[38] For each of the simulated pumping tests, the interpreted transmissivity $T_{i}(t)$ is estimated according to equation (4) using drawdown data from an observation point located at a distance $r_{o}=I / 8$ from the pumping well. As in the previous estimate, the $T_{i}(t)$ relation is converted into
$T_{i}(r)$ using equation (16). The interpreted transmissivity $T_{i}(r)$ is then compared to the transmissivity geometric mean $T_{g}(r)$ at $r=I, 2 I, 4 I$, and $8 I$. Figure 8 compares $T_{i}(r)$ and $T_{g}(r)$ for the case of $I=8$ and $\sigma_{Y}^{2}=1$. Each plot also shows the Pearson's correlation coefficient between the two sets of data $T_{i}(r)$ and $T_{g}(r)$. The results show good agreement between the two sets of data $T_{i}(r)$ and $T_{g}(r)$ for all distances considered, with the Pearson's coefficients ranging from 0.921 to 0.985 . Figure 9 compares the $T_{i}(r)$ to $T_{g}(r)$ for the case of $I=8$ and $\sigma_{Y}^{2}=2$. The correlation coefficients vary from 0.863 to 0.965 . These values are slightly lower than the case with $\sigma_{Y}^{2}=1$, however, the agreement between the two curves remains high.

[39] In summary, using the methodology proposed in this paper one would obtain a picture of the radially integrated transmissivity field as a function of distance (e.g., Figure 6). Through further analysis, this information can potentially be used to infer the spatial structure of the transmissivity field and, subsequently, in generating conditional $T$ fields that honor the pumping test interpretation results. For illustration, the relationship between the integral scale and variance on the interpreted transmissivity values can be qualitatively assessed from Figure 6. The integral scale of the transmissivity field influences the distance beyond which the interpreted transmissivity
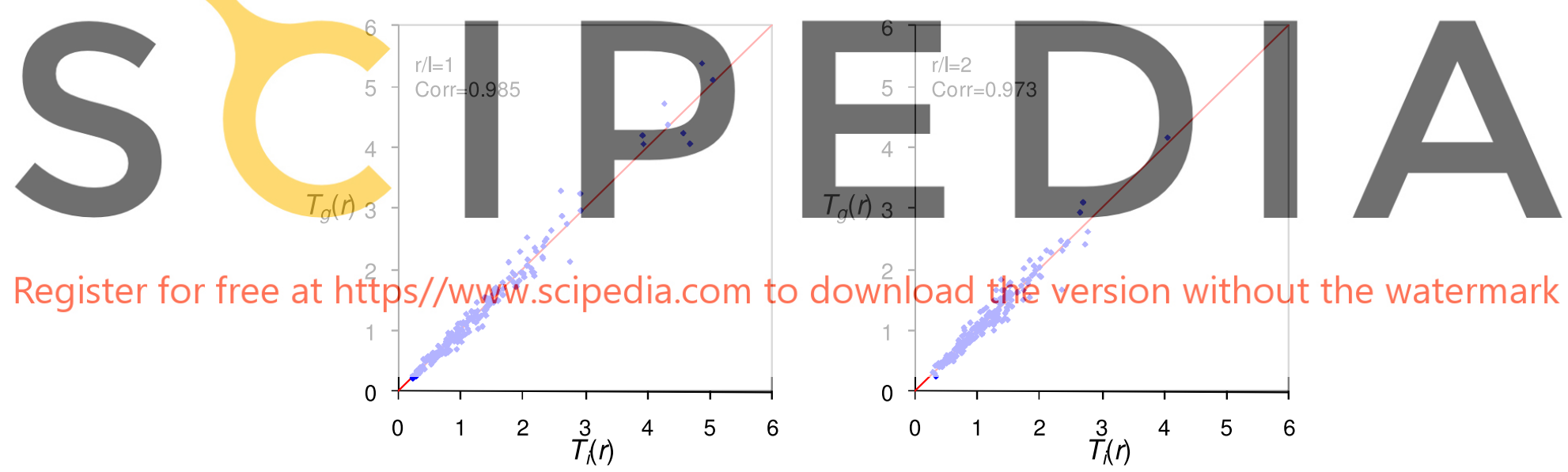

Register for free at https//wwwis.scipedia.com to download the version without the watermark
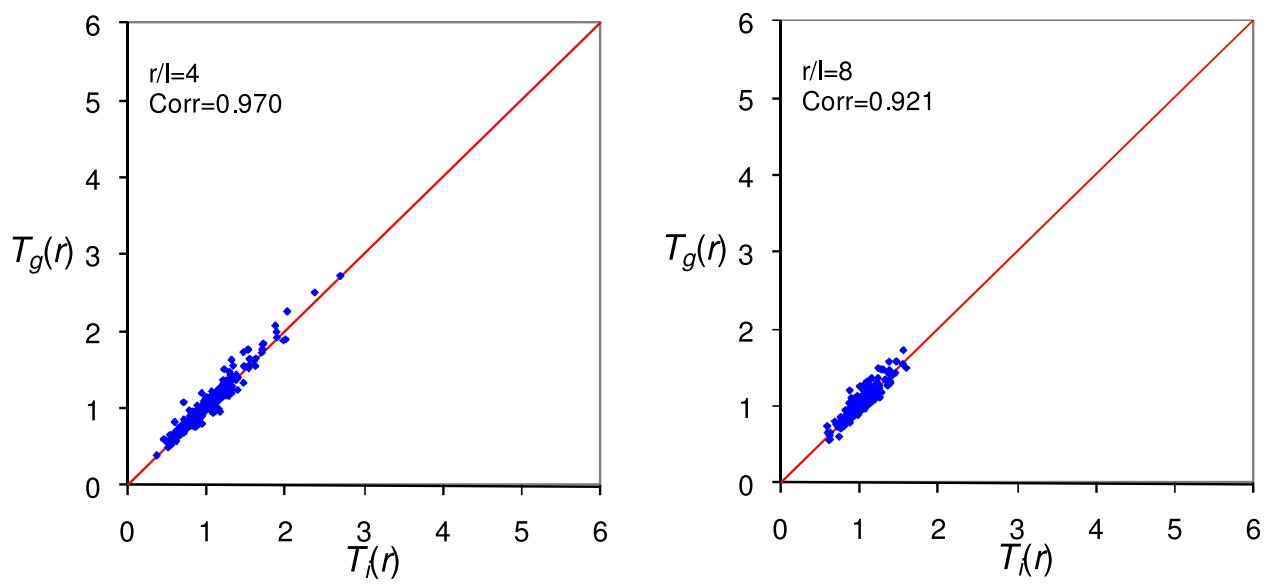

Figure 8. Comparison of the interpreted transmissivity and the geometric mean of the transmissivity for different distances from the well and for $I=8$ and $\sigma_{Y}^{2}=1$. Each point corresponds to one individual simulation, for a total of 200 . 

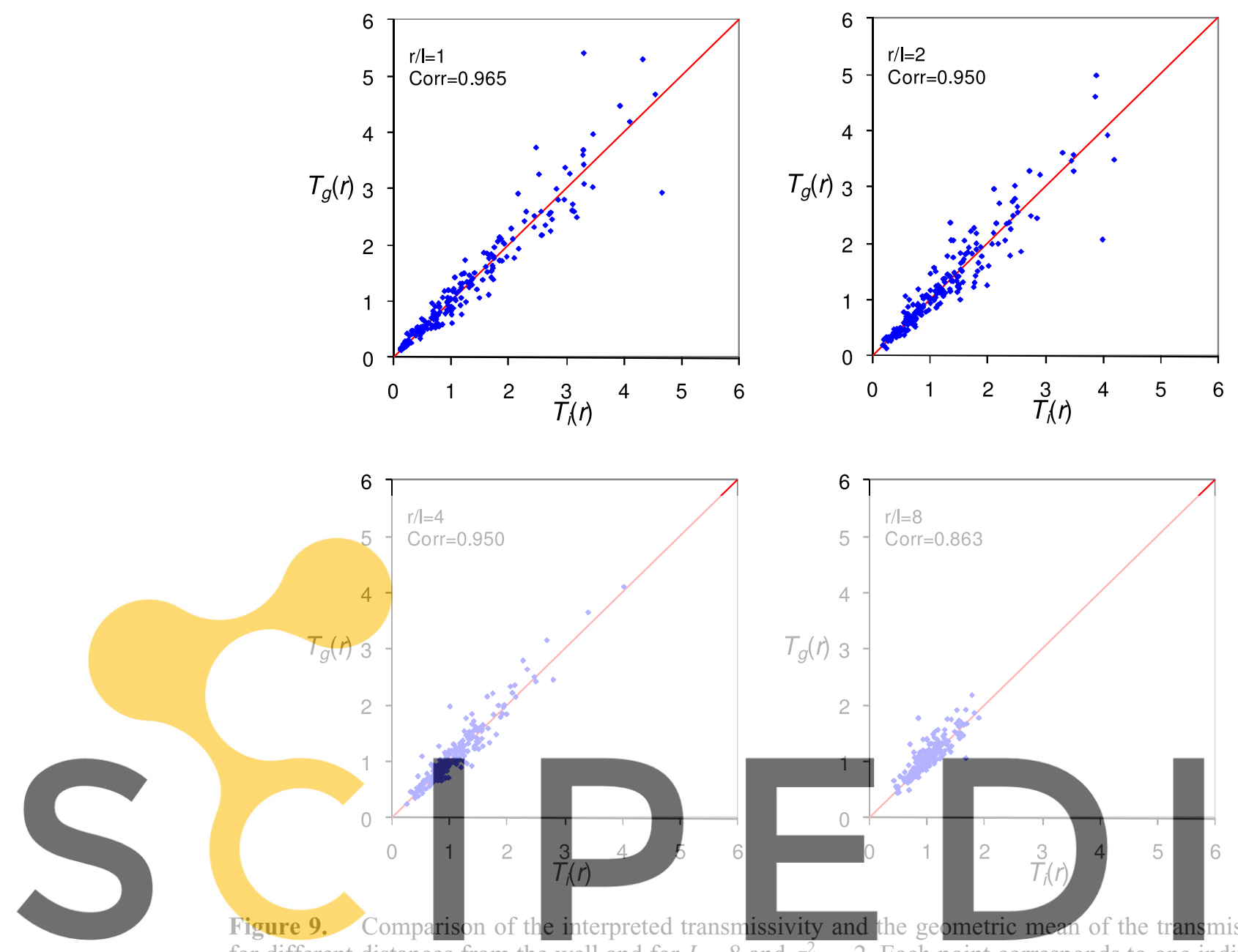

for different distances from the well and for $I=8$ and $\sigma_{Y}^{2}=2$. Each point corresponds to one individual simulation, for a total of 200.

Register for free at https//www.scipedia.com to other hand, the $\ln (T)$ variance influences the magnitude of the variations in the interpreted transmissivity (vertical axis of Figure 6). When multiple pumping tests are available, higher-reliability estimates of the variance and integral scale may be obtained by jointly evaluating the interpreted $T$ plots, each determined from an individual pumping test using the outlined procedure.

\section{Conclusions}

[40] Although heterogeneity of flow parameters is a salient characteristic of almost all natural geologic systems, pumping test data are widely analyzed using methods that are based on the assumption of aquifer homogeneity. This paper describes a new method for the analysis of pumping tests in heterogeneous confined aquifers that can provide information about the spatial variability of flow parameters rather than estimating a single representative value of the perturbed aquifer. The method uses the ratio of the drawdown to the drawdown derivative at a single point in time to estimate the transmissivity and storativity at the considered time. This is repeated over time yielding time-dependent interpreted flow parameters, $T_{i}(t)$ and $S_{i}(t)$. The time-dependent interpreted transmissivity is then expressed as a function of radial distance from the well.

\section{download the version without the watermark [41] The method is applied to randomly generated multi- variate Gaussian $\ln (T)$ fields. Results show that for $\ln (T)$ var-} iances up to 2 and for observation points located up to $r=I$ from the pumping well, the interpreted transmissivity $T_{i}(r)$ estimated with the proposed procedure is consistently in good agreement with the geometric mean of the transmissivity $T_{g}(r)$ computed over a circular area of the aquifer centered around the pumping well. As the separation distance between the observation point and the pumping well increases, $T_{i}(r)$ can still provide partial information about the radial trend of $T_{g}(r)$. However, estimates of transmissivity in the immediate vicinity of the well cannot be inferred as a result of the delay in drawdown response at the observation point.

[42] The interpreted storativity $S_{i}(t)$ is shown to depend on the point-to-point connectivity of the transmissivity field, consistent with previous studies. $S_{i}(t)$ is close to the actual value at early times. At later times, storativity is shown to be dependent on the connectivity of the $T$ field. Moreover, $S_{i}(t)$ decreases as $T_{i}(t)$ increases, and vice versa.

[43] In summary, the results presented in this paper show that additional information about the spatial variability of transmissivity can be derived from time-drawdown data. While conventional single-well pumping test interpretation methods use time-drawdown data to estimate a single representative estimate of the transmissivity, we propose in this paper a method based on time-drawdown data and its 
time derivative that yields an estimate of the geometric mean of transmissivity defined over a continuously increasing circle centered at the pumping well.

[44] Acknowledgments. N. Copty acknowledges the financial support provided by the Bogazici University Research Fund (BAP), Project 06M107. Paolo Trinchero acknowledges the financial support provided by the Torres Quevedo Programme of the Spanish Ministry of Science and Innovation (PTQ-09-01-00695). X. Sanchez-Vila acknowledges the financial support provided by the Spanish CICYT (project PARATODO).

\section{References}

Bellin, A., and Y. Rubin (2004), On the use of peak concentration arrival times for the inference of hydrogeological parameters, Water Resour. Res., 40, W07401, doi:10.1029/2003WR002179.

Bohling, G. C., X. Zhan, J. J. Butler Jr., and L. Zheng (2002), Steady shape analysis of tomographic pumping tests for characterization of aquifer heterogeneities, Water Resour. Res., 38(12), 1324, doi:10.1029/ 2001WR001176

Bourdet, D., J. A. Ayoub, and Y. M. Pirard (1989), Use of pressure derivative in well-test interpretation, Soc. Pet. Eng. Form. Eval., 4, 293-302.

Butler, J. J., C. D. McElwee, and G. C. Bohling (1999), Pumping tests in networks of multilevel sampling wells: Motivation and methodology, Water Resour. Res., 35(11), 3553-3560, doi:10.1029/1999WR900231.

Butler, J. J., Jr., J. M. Healey, G. W. McCall, E. J. Garnett, and S. P. Loheide II (2002), Hydraulic tests with direct-push equipment, Ground Water, 40(1), 25-36.

Butler, J. J., Jr., P. Dietrich, V. Wittig, and T. Christy (2007), Characterizing hydraulic conductivity with the direct-push permeameter, Ground Water, 45(4), 409-419.

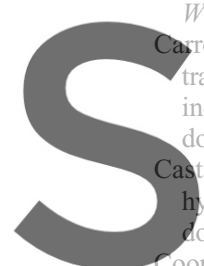
era, J., and S. P. Neuman (1986), Estimation of aquifer parameters under ransient and steady state conditions: 1. Maximum likelihood method incorporating prior information, doi:10.1029/WR022i002p00199. agna, M., and A. Bellin (2009), A Bayesian approach for inversion of i: 10.1029/2008WR007078 oper, H., and C. Jacob (1946), A generalized graphical method for evaluating formation constants and summarizing well-field history, Eos Trans. $A G U, 27(4), 526-534$

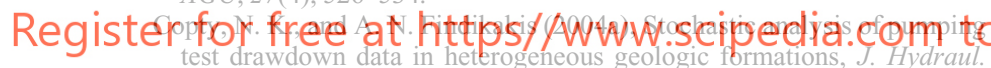
Res., 42, 59-67.

Copty, N. K., and A. N. Findikakis (2004b), Bayesian identification of the local transmissivity using time-drawdown data from pumping tests, Water Resour. Res., 40, W12408, doi:10.1029/2004WR003354.

Copty, N. K., P. Trinchero, M. S. Sarioglu, A. N. Findikakis, and X. Sanchez-Vila (2008), Influence of heterogeneity on the interpretation of pumping test data in leaky aquifers, Water Resour. Res., 44, W11419, doi:10.1029/2008WR007120.

Dagan, G. (1982), Analysis of flow through heterogeneous random aquifers: 2. Unsteady flow in confined formations, Water Resour. Res., 18(5), 1571-1585, doi:10.1029/WR018i005p01571.

Dagan, G., S. C. Lessoff, and A. Fiori (2009), Is transmissivity a meaningful property of natural formations? Conceptual issues and model development, Water Resour. Res., 45, W03425, doi:10.1029/2008WR007410.

de Marsily, G., J. P. Delhomme, F. Delay, and A. Abuoro (1999), 40 years of inverse problems in hydrogeology, Earth Planet. Sci., Ser. No. 329, 73-87.

Desbarats, A. J. (1992), Spatial averaging of transmissivity in heterogeneous fields with flow toward a well, Water Resour. Res., 28(3), 757-767, doi:10.1029/91WR03099.

Dietrich, P., and C. Leven (2005), Direct Push Technologies. In Groundwater Geophysics, edited by R. Kirsch, pp. 321-340, Springer, Berlin, Germany.

Dietrich, P., J. J. Butler Jr., and K. Faiss (2008), A rapid method for hydraulic profiling in unconsolidated formations, Ground Water, 46(2), 323-328.

Fernàndez-Garcia, D., P. Trinchero, and X. Sanchez-Vila (2010), Conditional stochastic mapping of transport connectivity, Water Resour. Res., 46, W10515, doi:10.1029/2009WR008533.

Fienen, M. N., T. Clemo, and P. K. Kitanidis (2008), An interactive Bayesian geostatistical inverse protocol for hydraulic tomography, Water Resour. Res., 44, W00B01, doi:10.1029/2007WR006730.
Finsterle, S., and M. Kowalsky (2008), Joint hydrological-geophysical inversion for soil structure identification, Vadose Zone J., 7, 287-293.

Firmani, G., A. Fiori, and A. Bellin (2006), Three-dimensional numerical analysis of steady state pumping tests in heterogeneous confined aquifers, Water Resour. Res., 42, W03422, doi:10.1029/2005WR004382.

Gelhar, L. W. (1993), Stochastic Subsurface Hydrology, 643 pp., PrenticeHall, Upper Saddle River, N. J.

Gottlieb, J., and P. Dietrich (1995), Identification of the permeability distribution in soil by hydraulic tomography, Inverse Probl., 11, 353-360, doi:10.1088/0266-5611.11/2/005.

Hantush, M. (1956), Analysis of data from pumping tests in leaky aquifers, Eos Trans. $A G U, 37(6), 702-714$.

Harbaugh, A., E. Banta, M. Hill, and M. McDonald (2000), Modflow2000: The US Geological Survey modular ground-water model-user guide to modularization concepts and the ground-water flow process, USGS Open-File Rep. 00-92, 121 pp.

Huang, H., B. X. Hu, X.-H. Wen, and C. Shirley (2004), Stochastic inverse mapping of hydraulic conductivity and sorption partitioning coefficient fields conditioning on nonreactive and reactive tracer test data, Water Resour. Res., 40, W01506, doi:10.1029/2003WR002253.

Illman, W. A., and S. P. Neuman (2001), Type-curve interpretation of a cross-hole pneumatic test in unsaturated fractured tuff, Water Resour. Res., 37(3), 583-604, doi:10.1029/2000WR900273.

Illman, W. A., X. Liu, and A. J. Craig (2007), Steady-state hydraulic tomography in a laboratory aquifer with deterministic heterogeneity: Multimethod and multiscale validation of hydraulic conductivity tomograms, J. Hydrol., 341(3-4), 222-234, doi:10.1016/j.jhydrol. 2007.05.011.

Knight, J. H., and G. J. Kluitenberg (2005), Analytical solutions for sensitivity of well tests to variations in storativity and transmissivity, $A d v$. Water Resour., 28, 1057-1075.

Köber, R., G. Hornbruch, C. Leven, L. Tischer, J. Grossmann, P. Dietrich, H. Weiss, and A. Dahmke. (2009), Evaluation of combined direct-push

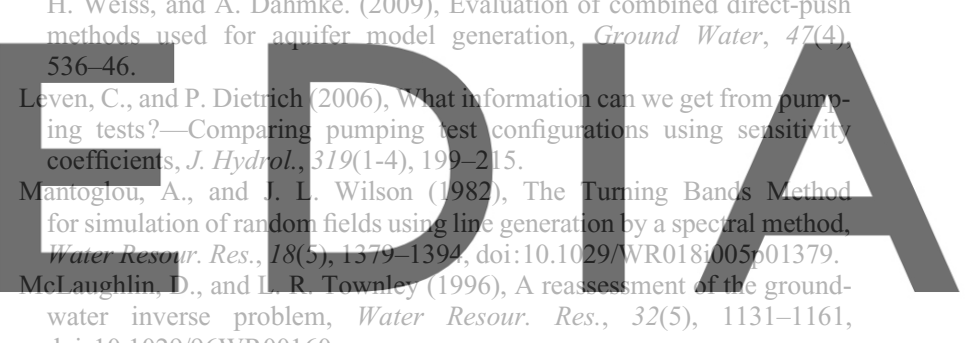

water inverse problem,
doi:10.1029/96WR00160.

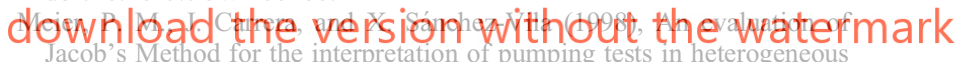 formations, Water Resour. Res., 34(5), 1011-1025, doi:10.1029/ 98WR00008}

Neuman, S. P., A. Guadagnini, and M. Riva (2004), Type-curve estimation of statistical heterogeneity, Water Resour. Res., 40, W04201, doi:10.1029/ 2003 WR002405.

Neuman, S. P., A. Blattstein, M. Riva, D. M. Tartakovsky, A. Guadagnini, and T. Ptak (2007), Type curve interpretation of late-time pumping test data in randomly heterogeneous aquifers, Water Resour. Res., 43, W10421, doi:10.1029/2007WR005871.

Oliver, D. S. (1993), The influence of nonuniform transmissivity and storativity on drawdown, Water Resour. Res., 29(1), 169-178, doi:10.1029/ 92WR02061.

Parker, R. L. (1994), Geophysical Inverse Theory, Princeton University Press, Princeton, N. J.

Ptak, T., M. Piepenbrink, and E. Martac. (2004), Tracer tests for the investigation of heterogeneous porous media and stochastic modeling of flow and transport-a review of some recent developments, J. Hydrol., 294(1-3), 122-163.

Riva, M., A. Guadagnini, J. Bodin, and F. Delay. (2009), Characterization of the hydrogeological experimental site of Poitiers (France) by stochastic well testing analysis, J. Hydrol., 369(1-2), 154-164.

Rubin, Y., and S. Hubbard, (2005), Water Sci. Technol. Library, Vol. 50, Hydrogeophysics, 523 pp., Springer, Berlin.

Sanchez-Vila, X., P. M. Meier, and J. Carrera (1999), Pumping tests in heterogeneous aquifers: An analytical study of what can be obtained from their interpretation using Jacob's Method, Water Resour. Res., 35(4), 943-952, doi:10.1029/1999WR900007.

Sanchez-Vila, X., A. Guadagnini, and J. Carrera (2006), Representative hydraulic conductivities in saturated groundwater flow, Rev. Geophys., 44(3), RG3002, doi:10.1029/2005RG000169. 
Serrano, S. E. (1997), The Theis solution in heterogeneous aquifers, Ground Water, 35(3), 463-467.

Slater, S. (2007), Near surface electrical characterization of hydraulic conductivity: from petrophysical properties to aquifer geometriesA review, Surv. Geophys. 28, 169-197, doi:10.1007/s10712-0079022-y.

Spane, F. A., and S. K. Wurstner (1993), DERIV : A Computer Program for calculating pressure derivatives for use in hydraulic test analysis, Groundwater, 31(5), 814-822.

Sun, N.-Z. (1994), Inverse Problems in Groundwater Modeling, 352 pp., Springer, Berlin.

Sun, N.-Z., and W. W.-G. Yeh (2007), Development of objective-oriented groundwater models: 1. Robust parameter identification, Water Resour. Res., 43, W02420, doi:10.1029/2006WR004887.

Theis, C. V. (1935), The relation between the lowering of the piezometric surface and the rate and duration of discharge of a well using groundwater storage, Eos Trans. AGU, 16, 519-524.

Trinchero, P., X. Sanchez-Vila, N. K. Copty, and A. N. Findikakis (2008a), A new method to interpret pumping tests in leaky aquifers, Ground Water, 46(1), 133-143.

Trinchero, P., X. Sanchez-Vila, and D. Fernandez-Garcia (2008b), Pointto-point connectivity, an abstract concept or a key issue for risk assessment studies?, Adv. Water Resour., 31(12), 1742-1753.
Walton, W. (1962), Selected analytical methods for well and aquifer evaluation, in Illinois State Water Survey, Bulletin No. 49, 81 pp., Urbana, Illinois.

Wu, C.-M., T.-C. J. Yeh, T. H. Lee, N.-S. Hsu, C. H. Chen, and A. F. Sancho (2005), Traditional aquifer tests: Comparing apples to oranges?, Water Resour. Res., 41(9), W09402, doi:10.1029/2004WR003717.

Yeh, T.-C. J., and S. Liu (2000), Hydraulic tomography: Development of a new aquifer test method, Water Resour. Res., 36(8), 2095-2105, doi:10.1029/2000WR900114.

Yin, D. T., and W. A. Illman (2009), Hydraulic tomography using temporal moments of drawdown recovery data: A laboratory sandbox study, Water Resour. Res., 45, W01502, doi:10.1029/2007WR006623.

Zhu, J., and T. J. Yeh (2005), Characterization of aquifer heterogeneity using transient hydraulic tomography, Water Resour. Res., 41, W07028, doi:10.1029/2004WR003790.

N. K. Copty, Institute of Environmental Sciences, Bogazici University, Istanbul, 34342 Turkey. (ncopty@boun.edu.tr)

X. Sanchez-Vila, Department of Geotechnical Engineering and Geosciences, Universitat Politecnica de Catalunya-BarcelonaTECH, Jordi Girona 31, E-08034 Barcelona, Spain.

P. Trinchero, AMPHOS 21 Consulting S.L., Passeig de García i Faria, 49-51, 1-1, E-08019 Barcelona, Spain. 